\title{
Pre-Clinical Evaluation of the Nanoliposomal antiPCSK9 Vaccine in Healthy Non-Human Primates
}

\author{
Amir Abbas Momtazi-Borojeni 1,2,3, Mahmoud R. Jaafari 1,4 , Maciej Banach 5,6 , Armita Mahdavi Gorabi ${ }^{7}$, \\ Hedayat Sahraei ${ }^{8}$ and Amirhossein Sahebkar $4,9,10, *$
}

1 Nanotechnology Research Center, Pharmaceutical Technology Institute, Mashhad University of Medical Sciences, Mashhad 9177948564, Iran; abbasmomtazi@yahoo.com (A.A.M.-B.); jafarimr@mums.ac.ir (M.R.J.)

2 Department of Medical Biotechnology, School of Medicine, Alborz University of Medical Sciences, Karaj 3149969415, Iran

3 Iran's National Elites Foundation, Tehran 9311114578, Iran

4 Biotechnology Research Center, Pharmaceutical Technology Institute, Mashhad University of Medical Sciences, Mashhad 9177948564, Iran

5 Department of Preventive Cardiology and Lipidology, Medical University of Lodz, 93-338 Lodz, Poland; maciej.banach@icloud.com

6 Cardiovascular Research Centre, University of Zielona Gora, 65-046 Zielona Gora, Poland

7 Research Center for Advanced Technologies in Cardiovascular Medicine, Tehran Heart Center, Tehran University of Medical Sciences, Tehran 1411713138, Iran; armitamahdavi61@gmail.com

8 Neuroscience Research Center, Baqiyatallah University of Medical Sciences, Tehran 9311114578, Iran; hsahraei1343@gmail.com

9 Applied Biomedical Research Center, Mashhad University of Medical Sciences, Mashhad 9177948564, Iran

10 School of Pharmacy, Mashhad University of Medical Sciences, Mashhad 9177948564, Iran

* Correspondence: sahebkara@mums.ac.ir or amir_saheb2000@yahoo.com

Citation: Momtazi-Borojeni, A.A.; Jaafari, M.R.; Banach, M.; Gorabi, A.M.; Sahraei, H.; Sahebkar, A. Pre-Clinical Evaluation of the Nanoliposomal antiPCSK9 Vaccine in Healthy Non-Human Primates. Vaccines 2021, 9, 749. https:// doi.org/10.3390/vaccines9070749

Academic Editor: Giuseppe Bardi

Received: 8 June 2021

Accepted: 4 July 2021

Published: 6 July 2021

Publisher's Note: MDPI stays neutral with regard to jurisdictional claims in published maps and institutional affiliations.

Copyright: (c) 2021 by the authors. Licensee MDPI, Basel, Switzerland. This article is an open access article distributed under the terms and conditions of the Creative Commons Attribution (CC BY) license (https:/ / creativecommons.org/licenses/by/ $4.0 /)$.

\begin{abstract}
Background: Our previous studies showed the safe preventive and therapeutic effects of immunization using the nanoliposomal antiPCSK9 vaccine called "Liposomal Immunogenic Fused PCSK9-Tetanus plus Alum adjuvant" (L-IFPTA), in mouse models of atherosclerosis. Here we aimed to ascertain the immunogenicity and safety of the L-IFPTA vaccine in a pre-clinical study in healthy non-human primates. Methods: Five male rhesus macaque monkeys were subcutaneously immunized with the L-IFPTA vaccine, four times with bi-weekly intervals. To evaluate immunogenicity, the plasma antiPCSK9 antibody in immunized monkeys was detected and quantified using the ELISA method. The functionality of the induced antiPCSK9 antibodies was determined by the PCSK9/LDLR in vitro binding assay kit. The safety of the vaccine was tested using the evaluation of several major circulating indicators including plasma lipid alterations, inflammatory biomarkers and organ injury biomarkers. Results: The resultant data indicated that the L-IFPTA vaccine significantly and highly induced the generation of functional and safe antiPCSK9 antibodies in immunized monkeys. Plasma levels of specific biomarkers indicating organ performance including creatinine, urea, uric acid, bilirubin, ALP, AS, ALT and TSH were not significantly altered. After immunization in healthy monkeys, non-prespecified endpoints (plasma levels of TC, LDL-C, VLDL-C and TG) were non-significantly reduced by $11.6 \pm 36 \% ; 16 \pm 28 \% ; 22 \pm 53 \%$ and $24 \pm 51 \%$, respectively, while HDL-C was slightly increased by $2 \pm 64 \%$. There were also no significant changes in plasma levels of pro- and anti-inflammatory biomarkers. Conclusion: The L-IFPTA vaccine could efficiently stimulate the host humoral immune response to produce active antibodies that inhibit plasma PCSK9 while not provoking systemic inflammation and not adversely affecting organ performance.
\end{abstract}

Keywords: nanoliposome; non-human primate; PCSK9; vaccine; cholesterol

\section{Introduction}

Currently, proprotein convertase subtilisin/kexin type 9 (PCSK9) inhibition is one of the most promising therapeutic approaches for lowering hypercholesterolemia and 
reducing the incidence of atherosclerotic cardiovascular disease (ACVD), particularly in patients with familial hypercholesterolemia and those experiencing statin resistance or intolerance [1-4]. PCSK9 is mainly produced by hepatocytes and secreted into the blood circulation where it can negatively regulate the protein expression of the low-density lipoprotein (LDL) receptor (LDLR) on the surface of hepatocytes. Circulating PCSK9 binds to the extracellular domain of LDLR, and then mediates the internalization and lysosomal degradation of LDLR, which leads to the lower clearance of LDL cholesterol (LDL-C) from the bloodstream and thus results in hypercholesterolemia [5].

Passive immunotherapy using monoclonal antibodies (mAbs) disrupting PCSK9/LDLR interaction provides the most advanced strategy for PCSK9 inhibition [6-9]. However, because of the short in vivo half-life of $\mathrm{mAbs}$, their long-term therapeutic application needs frequent administration (once or twice/month) at relatively high doses that might not be affordable in many countries [10-12]. Moreover, the use of mAbs may be limited by tolerability problems and possible induction of host anti-mAb antibodies [11-13]. The active vaccination using PCSK9 peptide antigens has represented a desirable alternative strategy to overcome the drawbacks of mAbs. This strategy can provide the same therapeutic effects as those achieved with mAbs but with fewer injections and lower doses, and less risk of promoting drug-neutralizing immune responses.

The adjuvanted anti-PCSK9 vaccines have been studied by different approaches, including human recombinant PCSK9 with a DNA oligonucleotide adjuvant [14]; human PCSK9 peptides displayed on "virus-like particles" [15-17], PCSK9-BSA peptide [18]; and peptides mimicking PCSK9 epitopes conjugated to a KLH-carrier developed by AFFiRiS group using the AFFITOPE ${ }^{\circledR}$ technology $[19,20]$. To date, only AFFiRiS group has tested the antiPCSK9 vaccine in a phase I human clinical trial; however, these results are not available yet.

During the recent few years, we have developed a new adjuvanted antiPCSK9 vaccine using a combination of the AFFITOPE ${ }^{\circledR}$ and nanoliposome technology [21]. This vaccine, termed "Liposomal Immunogenic Fused PCSK9-Tetanus plus Alum adjuvant" (L-IFPTA) has been shown to have efficacy in various animal models [21-24]. The early study revealed that the L-IFPTA vaccine could effectively induce safe and long-term (one year) production of antiPCSK9 antibodies in BALB/c mice [21]. Further studies on C57BL/6 mice with severe hypercholesterolemia and atherosclerosis indicated that the L-IFPTA vaccine could efficiently exert long-lasting therapeutic [22] and preventive [25] effects against hypercholesterolemia and atherosclerosis. Such promising results of murine studies suggested L-IFPTA as an appropriate candidate for testing in clinical trials. To this end, in the present study, we aimed to ascertain the immunogenicity and safety of the LIFPTA vaccine in healthy non-human primates, which have a high physiological similarity to humans.

\section{Methods}

\subsection{The L-IFPTA Vaccine Formulation}

The L-IFPTA vaccine was developed as explained in detail in our previous publication [21]. Briefly, to prepare L-IFPTA vaccine formulation, an immunogenic peptide construct embracing PCSK9 and tetanus epitopes (Table 1) termed "Immunogenic Fused PCSK9-Tetanus" (IFPT) was attached to the surface of already synthesized liposome nanoparticles. Lipid formulation containing 1,2-Dimyristoyl-sn-glycero-3-phosphorylcholine (DMPC); 1,2-Dimyristoyl-sn-glycero-3-phosphorylglycerol (DMPG); and cholesterol (Avanti Polar Lipid; Alabaster, AL, USA) was particlized to homogeneous nanoliposomes, using the lipid-film hydration followed by the extrusion method. The IFPTA peptide construct (synthesized by ChinaPeptides Co., Ltd., Shanghai, China) was attached to the surface of the prepared nanoliposome particles (termed L-IFPT), using DSPE-PEG-Mal (1,2-distearoylsn-glycero-3-phosphoethanolamine- $\mathrm{N}$-[maleimide(PEG)-2000]) lipid linker (Lipoid GmbH, Ludwigshafen, Germany). The conjugation between the peptides and liposome parti- 
cles was confirmed using the thin layer chromatography (TLC); tricin-SDS-PAGE; and high-performance liquid chromatography (HPLC) as explained previously [21].

Table 1. Sequence of the immunogenic peptides used in the present study.

\begin{tabular}{ccc}
\hline Peptide Name & Sequence & Immunogenicity \\
\hline PCSK9 & S-I-P-W-N-L-E-R-I-T-P-V-R & B cell epitope \\
Tetanus & A-Q-Y-I-K-A-N-S-K-F-I-G-I-T-E-L & T cell epitope \\
IFPT & $*$ CGGGSIPWNLERITPVRKKAQYIKANSKFIGITEL & \\
\hline${ }^{*}$ The bold amino acid codes exhibit linker sequences. IFPT; Immunogenic Fused PCSK9-Tetanus.
\end{tabular}

To purify peptide-conjugated liposome nanoparticles, the free peptides were eliminated using dialysis membrane sucks with 12-14 KD MWCO (Merck, Darmstadt, Germany; D6191-25EA).

Physical characters of L-IFPT nanoparticles, including particle size, surface charge, and polydispersity index (PDI) were measured using the dynamic light scattering (DLS) technique on a Zetasizer (Nano-ZS, Malvern, UK). To evaluate the stability, physical characters of L-IFPT nanoparticles stored at $4{ }^{\circ} \mathrm{C}$ and $25{ }^{\circ} \mathrm{C}$ have been measured monthly for up to six months. The vaccine formulation was completed by mixing the prepared L-IFPT nanoparticles to $0.4 \%$ Alum adjuvant (Sigma-Aldrich) at a 1:1 (v:v) ratio, which thereafter is called L-IFPTA.

\subsection{Macaques Monkey Vaccination}

Rhesus macaque monkeys (Macaca mulatta) were obtained from the animal house of the Neuroscience Research Center of the Baqiyatallah University of Medical Sciences (Tehran, Iran). All studies on macaques were performed by an expert veterinary physician in accordance with the animal welfare guidelines approved by the Institutional Ethics Committee and Research Advisory Committee of Mashhad University of Medical Sciences, Mashhad, Iran (IR.MUMS.PHARMACY.REC.1397.113). Five 7-9 year-old-male macaque monkeys (weight: 8-11 kg) were subcutaneously vaccinated four times at 2-week intervals with the L-IFPTA formulation containing $100 \mu \mathrm{g}$ peptide per injection. Venous blood sampling was done prior to the vaccination at week 0 and two weeks following each vaccination up to week 8 (Figure 1). Redness, swelling or induration at the vaccination site, food intake and body weight, as well as malaise, fever, shivering or rash were checked during the vaccination period.

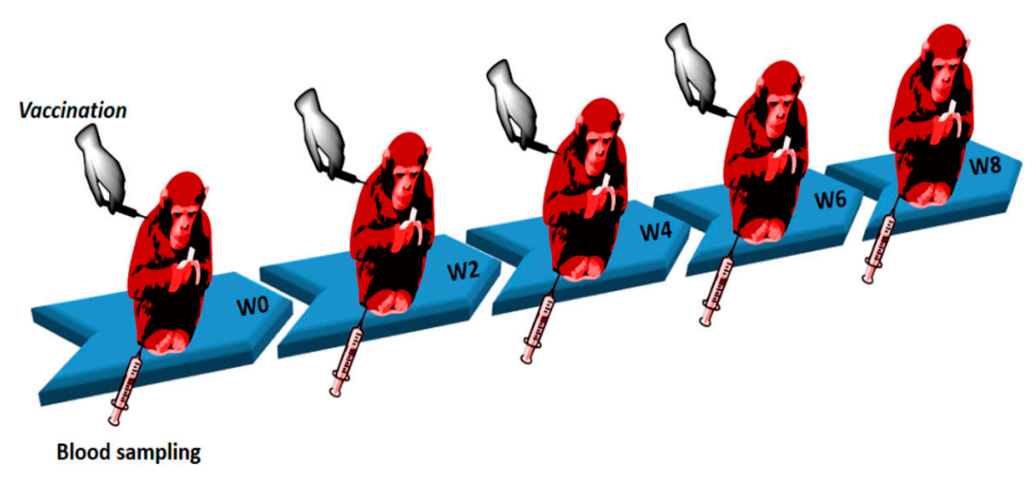

Figure 1. Schematic diagram of vaccination and blood sampling schedule.

\subsection{Characterization of Antibody Responses}

To determine the immunogenicity of the L-IFPTA vaccine, titers of antiPCSK9-specific IgG were measured by ELISA technique, using PCSK9 peptide (ChinaPeptides Co., Ltd., Shanghai, China) as the antigen [21]. Detection was performed by HRP-conjugated antimonkey IgG (Sigma Aldrich; dilution 1:1000), incubated for $1 \mathrm{~h}$ at $37^{\circ} \mathrm{C}$ followed by the addition of the substrate TMB (3,3',5,5'-tetramethylbenzidine) (Sigma-Aldrich; $15 \mathrm{~min}$ 
at RT). The optical density (OD) at $450 \mathrm{~nm}$ was measured with a microwell plate reader (BioTek, Synergy 2 plate reader, Winooski, VT, USA), and the titers were considered as the dilution factor corresponding to $50 \%$ of the maximal optical density $\left(\mathrm{OD}_{\max } / 2\right)$.

\subsection{Evaluating the Effect of Induced antiPCSK9 Antibodies on PCSK9/LDLR Interaction In Vitro}

To evaluate the ability of vaccine-produced antibodies for hampering the PCSK9/LDLR interaction, an in vitro binding assay kit (CircuLex ${ }^{\mathrm{TM}}$ PCSK9/LDLR, Cy- 8150, MBL, Woburn, MA, USA) was used according to the manufacturer's manual. The principle of the assay is based on the in vitro binding between recombinant PCSK9 and recombinant epidermal growth factor-like repeat (EGF-A) domain of LDLR, which would be suppressed in the presence of plasma containing antiPCSK9 antibodies. In this method, the higher amount of PCSK9 binding to LDLR is correlated with higher ELISA OD, in which at the presence of anti-PCSK9 antibody this interaction is suppressed, and, consequently, ELISA OD is reduced. The detailed protocol is clearly described in our previous publication [21].

\subsection{The Plasma Lipid Assay}

Plasma levels of total cholesterol (TC), VLDL-C, high-density lipoprotein cholesterol (HDL-C), and triglyceride (TG) in vaccinated monkeys were measured using the Biosystems kits (Biosystems S.A., Barcelona, Spain).

\subsection{The Inflammatory Biomarker Assay}

In order to assess systemic inflammation upon vaccination in macaque monkeys, a panel of plasma inflammatory markers, including L- $1 \alpha$, IL-1 $\beta$, IL-2, IL-4, IL-6, IL-8, IL-10, VEGF, EGF, TNF- $\alpha$, IFN- $\gamma$, hs-CRP, and MCP-1, were quantified using a cytokine array biochip (Randox, London, UK).

\subsection{The Assessment of Organ Injury Biomarkers}

As indicators for toxicity, plasma levels of biomarkers of organ injury, including creatinine, urea, uric acid, alkaline phosphatase (ALP), aspartate transaminase (AST), alanine transaminase (ALT), bilirubin, and thyroid-stimulating hormone (TSH) were measured in vaccinated macaque monkeys, using the Biosystems kits (Biosystems S.A., Barcelona, Spain) according to the manufacturer's manual.

\subsection{Statistical Analysis}

An unpaired two-tailed Student's $t$-test was carried out to define the significance of difference among groups (Graph Pad Prism Software, version 7, San Diego, CA, USA). Data were reported as mean $\pm \mathrm{SD}$. Values with $p<0.05$ were regarded to be statistically significant.

\section{Results}

\subsection{Characterization and Stability of L-IFPT Nanoparticles}

Physical properties of the free and IFPT-linked liposomal nanoparticles are summarized in Table 2. The size range of liposome nanoparticles was distributed from $130 \mathrm{~nm}$ to $160 \mathrm{~nm}$ in diameter, in which PDI was less than 0.01 , indicating nanoparticles with high homogeneity. The prepared nanoliposomes also showed a negative surface charge. The physical properties of L-IFPT nanoparticles, including the size (Figure 2A) and the surface charge (Figure 2B), showed no significant changes after 6 months of storage at $4{ }^{\circ} \mathrm{C}$, whereas significant changes were observed after 3 months at $25^{\circ} \mathrm{C}$, when compared to the baseline time point. Our results indicate that L-IFPT nanoparticles are stable for at least 6 months at the refrigerator temperature $\left(4^{\circ} \mathrm{C}\right)$ and for 3 months at room temperature $\left(25^{\circ} \mathrm{C}\right)$. 
Table 2. Physical properties of nanoliposomal formulations.

\begin{tabular}{cccc}
\hline Formulation & $\begin{array}{c}\text { Z-Average }(\mathbf{n m}) \\
{[\text { Mean } \pm \text { SD, } \boldsymbol{n}=3]}\end{array}$ & $\begin{array}{c}\text { Zeta Potential }(\mathbf{m V}) \\
{[\text { Mean } \pm \text { SD, } \boldsymbol{n}=3]}\end{array}$ & $\begin{array}{c}\text { PDI * } \\
\text { [Mean } \pm \text { SD, } \boldsymbol{n}=\mathbf{3}]\end{array}$ \\
\hline $\begin{array}{c}\text { The } \\
\text { free-nanoliposome }\end{array}$ & $134 \pm 5$ & $-41 \pm 2$ & $0.01 \pm 0.001$ \\
\hline $\begin{array}{c}\text { The IFPT } \\
\text { linked-nanoliposome }\end{array}$ & $159 \pm 8$ & $-28 \pm 3$ & $0.02 \pm 0.008$ \\
\hline
\end{tabular}

*PDI; Polydispersity index.
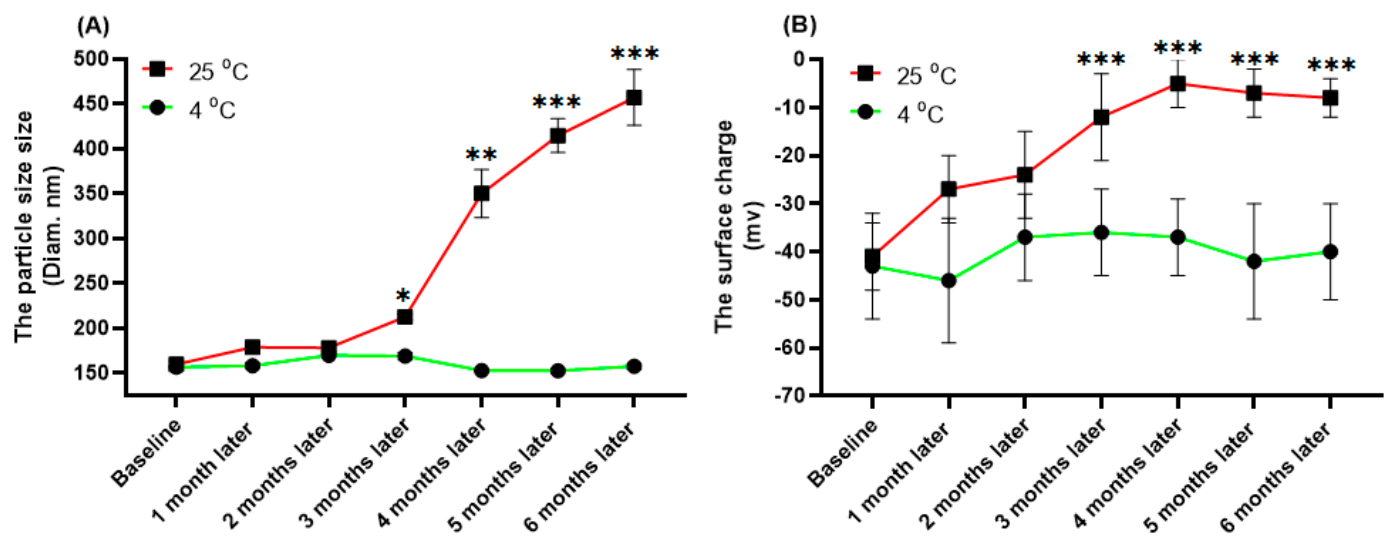

Figure 2. Evaluating the stability of L-IFPT nanoparticles by measuring changes of physical characteristics at $4{ }^{\circ} \mathrm{C}$ and $25^{\circ} \mathrm{C}$ for 6 months. The physical characteristics of L-IFPT nanoparticles, including the size (A) and the surface charge (B), were not significantly changed for up to 6 months of storage at the $4{ }^{\circ} \mathrm{C}$, while those were significantly increased after 3 months of storage at $25^{\circ} \mathrm{C}$, when compared to the baseline time point. Values are means $\pm \mathrm{SD}(n=3)$. Statistical differences at $p$-values less than 0.05 were considered to be significant. ${ }^{*}{ }^{* *}$, and ${ }^{* * *}$ signs show $p=0.03, p=0.01$, and $p<0.01$, respectively.

\subsection{The L-IFPTA Vaccine Is Immunogenic in the Rhesus Macaque}

Titer analyses over time indicated that the L-IFPTA vaccine was able to significantly promote a humoral immune response against PCSK9 in rhesus macaque monkeys (Figure 3A). AnitPCSK9 IgG antibodies were significantly raised and reached a maximum level at the mean titer of 1: 19,460 \pm 1726 (ODmax/2) at week 8, following three boosters after the prime immunization (Figure $3 \mathrm{~B}$ ).

(A)

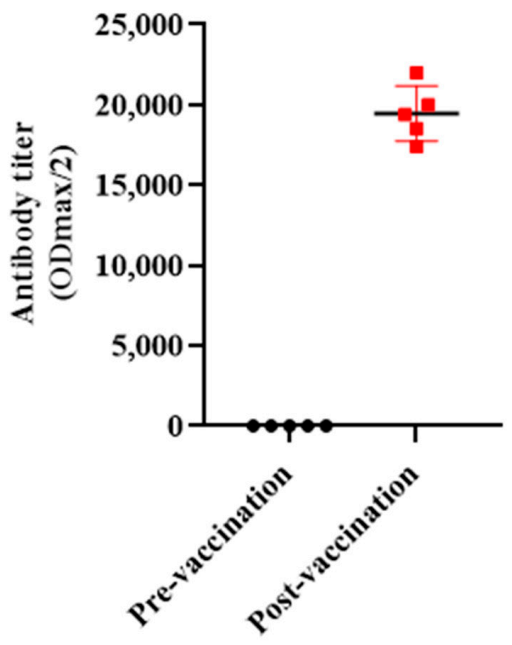

(B)

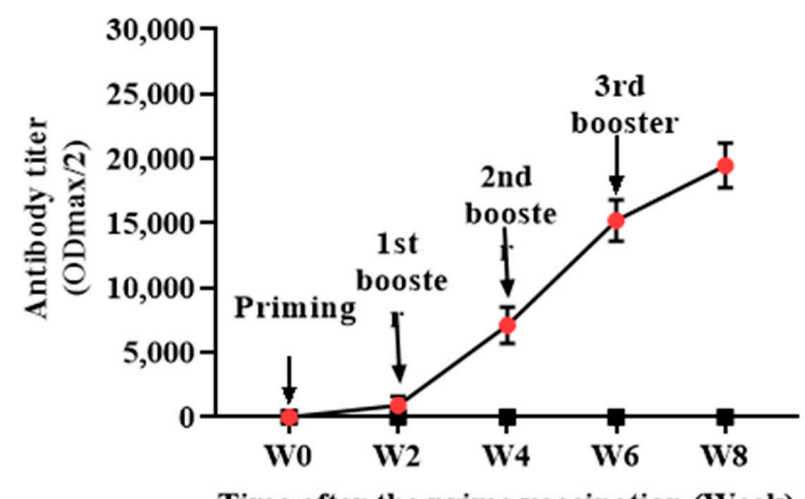

Time after the prime vaccination (Week)

Figure 3. (A) Antibody titer (ODmax/2) against PCSK9 in vaccinated monkeys at pre-vaccination (W0) and post-vaccination (W8) time-points. (B) The exponential increase in antiPCSK9 antibody titer (ODmax/2) over 8 weeks post prime vaccination, generated upon 4 vaccinations in a biweekly interval (signed by arrows). Values are means $\pm \operatorname{SD}(n=5)$. 


\subsection{Vaccine-Induced antiPCSK9 Antibodies Interfered with PCSK9 Function}

To evaluate whether the vaccine-induced antiPCSK9 antibodies are able to inhibit PCSK9 activity, the in vitro PCSK9/LDLR interaction was assayed in the presence of plasma samples isolated from vaccinated healthy monkeys at week 8 . It was found that the post-vaccination plasma samples containing antiPCSK9 antibodies could significantly diminish PCSK9 binding to LDLR by $-33 \pm 7 \%$ when compared to pre-vaccination plasma samples (Figure 4). The resultant data indicated that L-IFPTA vaccine could provoke the production of antiPCSK9 antibodies in the rhesus macaques, which blocked the PCSK9 activity through targeting EGF-A binding domain, thereby, suppressing PCSK9-mediated LDLR degradation.

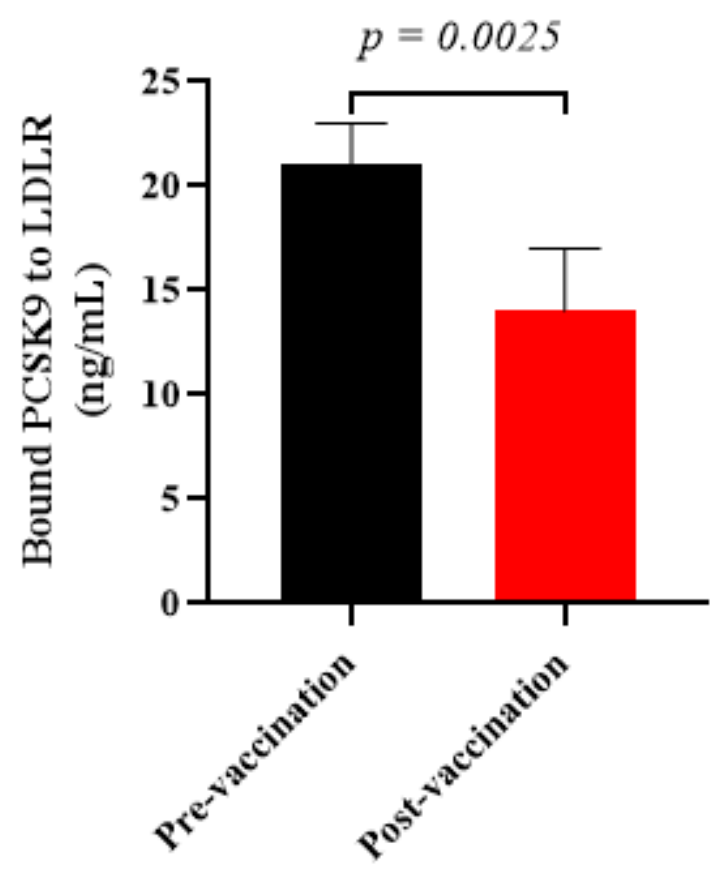

Figure 4. In vitro PCSK9/LDLR binding assay. Vaccine-produced antiPCSK9 antibodies suppress the interaction of PCSK9 and LDLR. A plasma sample of monkeys at post-vaccination time-point (W8) could reduce PCSK9 binding to LDLR by $-33 \pm 7 \%$, when compared with a plasma sample of pre-vaccination ones (W0). Values are means $\pm \mathrm{SD} ; n=3$ replicates of the pooled samples of 5 monkeys. The significance compared to pre-vaccination values was analyzed by an unpaired two-tailed Student's $t$-test. Statistical differences at $p$-values less than 0.05 were considered to be significant.

\subsection{The L-IFPTA Vaccine Did Not Cause Organ Injury in the Rhesus Macaque}

Plasma levels of specific indicators of the organ injury, including creatinine, urea, and uric acid (for kidneys); ALP, AST, ALT, and bilirubin (for the liver); as well as TSH (for thyroid glands), were not significantly changed in the vaccinated monkeys (Table 3).

Table 3. Plasma levels of the organ injury indicators in monkeys at pre- and post-vaccination time-points.

\begin{tabular}{|c|c|c|c|c|c|c|c|c|}
\hline Time-Point & $\begin{array}{c}\text { Creatinine } \\
(\mathrm{mg} / \mathrm{dL}, \\
\text { Mean } \pm \mathrm{SD})\end{array}$ & $\begin{array}{c}\text { Urea } \\
(\mathrm{mg} / \mathrm{dL}, \\
\text { Mean } \pm \mathrm{SD})\end{array}$ & $\begin{array}{c}\text { Uric Acid } \\
(\mathrm{mg} / \mathrm{dL}, \\
\text { Mean } \pm \mathrm{SD})\end{array}$ & $\begin{array}{c}\text { Bilirubin } \\
(\mathrm{mg} / \mathrm{dL}, \\
\text { Mean } \pm \mathrm{SD})\end{array}$ & $\begin{array}{c}\text { ALP } \\
(\mathrm{mg} / \mathrm{dL}, \\
\text { Mean } \pm \mathrm{SD})\end{array}$ & $\begin{array}{c}\text { AST } \\
(\mathrm{mg} / \mathrm{dL}, \\
\text { Mean } \pm \mathrm{SD})\end{array}$ & $\begin{array}{c}\text { ALT } \\
(\mathrm{mg} / \mathrm{dL}, \\
\text { Mean } \pm \mathrm{SD})\end{array}$ & $\begin{array}{c}\text { TSH } \\
(\mathrm{mg} / \mathrm{dL}, \\
\text { Mean } \pm \text { SD })\end{array}$ \\
\hline Pre-immunization & $1.2 \pm 0.2$ & $30.8 \pm 7$ & ND & ND & $2311 \pm 1121$ & $32 \pm 13$ & $2.8 \pm 1.5$ & $0.9 \pm 0.2$ \\
\hline Post-immunization & $1.1 \pm 0.1$ & $48 \pm 14$ & ND & ND & $1876 \pm 1125$ & $25 \pm 14$ & $2.4 \pm 1.1$ & $1 \pm 0.3$ \\
\hline Mean difference & NSD & NSD & - & - & NSD & NSD & NSD & NSD \\
\hline
\end{tabular}

Abbreviations: Alkaline phosphatase (ALP), Aspartate transaminase (AST), Alanine transaminase (ALT), Not defined (ND), NSD; No significant difference (NSD), Standard deviation (SD), Thyroid-stimulating hormone (TSH). 


\subsection{The L-IFPTA Vaccine Did Not Change Levels of Plasma Lipids in the Rhesus Macaque}

When compared the lipid profile in healthy monkeys at pre- and post-vaccination time-points, it was found that plasma levels of TC, LDL-C, VLDL-C, and TG were nonsignificantly $(p>0.05)$ decreased in vaccinated monkeys by $11.6 \pm 36 \%, 16 \pm 28 \%, 22 \pm 53$, and $24 \pm 51 \%$, respectively, while HDL-C was slightly increased by $2 \pm 64 \%(p>0.05)$ (Figure 5).

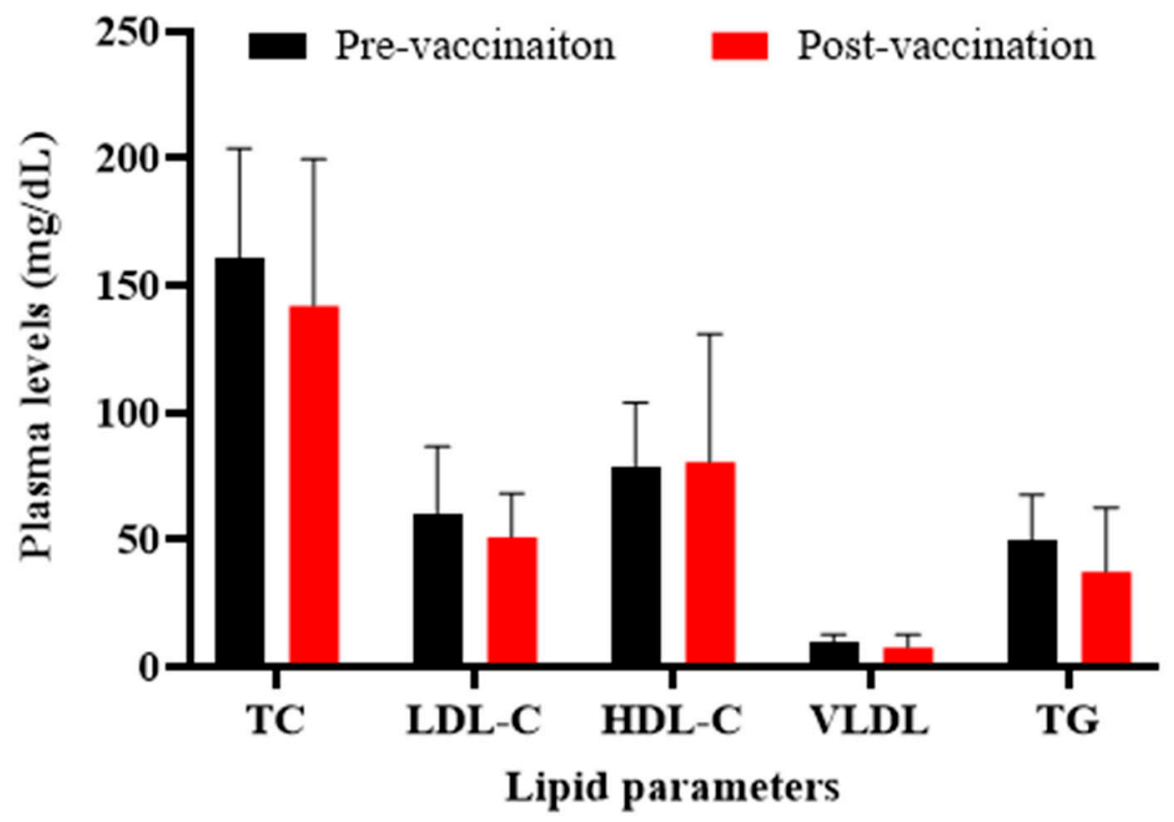

Figure 5. Plasma levels of lipid parameters in monkeys at pre- (W0) and post-vaccination (W0) time-points. There are no significant changes in plasma lipids levels, before and after vaccination, in the monkeys. Values are means $\pm \mathrm{SD}(n=5)$. The two-tailed Student's $t$-test was used to evaluate the presence of significant differences between values of pre- and post-vaccination time-points. Statistical differences at $p$-values less than 0.05 were considered to be significant.

\subsection{The L-IFPTA Vaccine Did Not Induce Systemic Inflammation in the Rhesus Macaque}

The results demonstrated that the L-IFPTA vaccine does not have significant effects on inflammation-related biomarkers including IL-1 $\alpha$, IL-1 $\beta$, IL-2, IL-4, IL-6, IL-8, IL-10, VEGF, EGF, TNF- $\alpha$, IFN- $\gamma$, hs-CRP, and MCP-1 in vaccinated monkeys (Table 4).

Table 4. Plasma levels of inflammatory biomarkers in monkeys at pre- and post-vaccination time-points.

\begin{tabular}{|c|c|c|c|c|c|c|c|c|c|c|c|c|c|}
\hline Time-Point & $\operatorname{IL1} \alpha$ & IL1 $\beta$ & IL2 & IL4 & IL6 & IL8 & IL10 & IFN $\gamma$ & TNF & EGF & VEGF & МСР-1 & CRP \\
\hline $\begin{array}{l}\text { Pre-vaccination } \\
\text { (pg/mL, mean } \pm \\
\text { SD) }\end{array}$ & ND & ND & ND & ND & $1 \pm 1.3$ & $6.6 \pm 8$ & $3.6 \pm 7$ & ND & ND & $2.6 \pm 6$ & ND & $171 \pm 31$ & $2.2 \pm 1.5$ \\
\hline $\begin{array}{c}\text { Post-vaccination } \\
\text { (pg/mL, mean } \pm \\
\text { SD) }\end{array}$ & ND & ND & ND & ND & ND & $25 \pm 7$ & ND & ND & ND & $62 \pm 37$ & ND & $156 \pm 76$ & $2 \pm 0.7$ \\
\hline Mean difference & - & - & - & - & NSD & NSD & NSD & - & - & NSD & - & NSD & NSD \\
\hline
\end{tabular}

IL: interleukin; IFN: interferon; TNF: tumor necrosis factor; EGF: epidermal growth factor; VEGF: vascular endothelial growth factor; MCP-1: monocyte chemoattractant protein-1; CRP: C-reactive protein; ND: not detected; NSD: no significant difference.

\section{Discussion}

Inducing the host's humoral immunity to produce functional and specific antibodies against a target molecule is an indispensable part of active immunotherapy. Here, we found that the L-IFPTA vaccine formulation could strongly induce the production of safe and functional antiPCSK9 antibodies in the rhesus macaques. Importantly, the vaccine- 
induced antibodies could significantly inhibit PCSK9 binding to the LDLR, confirming their functionality. This intervention was also safe, without any observed adverse effects.

These findings support our previous studies on BALB/c and C57BL/ 6 mice immunized by the L-IFPTA vaccine that showed similar results $[21,22,26,27]$. Of note, provoking antibody production in the two different species, rodents and non-human primates, is not surprising. This is because the immunogenic peptide antigen used in the formulation of the L-IFPTA vaccine [21] exploits AFFITOME ${ }^{\circledR}$ technology that uses mimotope antigens with closely identical sequences between species and can thus show immunogenicity in various species [28].

Notably, despite the previously observed efficient therapeutic $[22,26]$ and preventive [27] effects of the L-IFPTA vaccine for reducing hypercholesterolemia and atherosclerotic plaque progression in hyperlipidemic C57BL/6 mice, the present study showed a non-significant reduction in serum lipid indices in healthy monkeys. Of note, another antiPCSK9 vaccine formulation prepared using virus-like particle technology was reported to decrease serum LDL-C in an approximately similar but significant percentage (by 10-15\%) in healthy non-human primates [16]. Besides normolipidemic state, such a non-significant lipid reduction in the current study can be due to the small sample size of our study; therefore, the L-IFPTA vaccine might exert a significant and even a higher lipid-lowering effect with increasing the sample size and more suitable subjects (hyperlipidemic with additional risk factors, similarly to previous studies with PCSK9 inhibition [29]. The modest association between the effects of the vaccine on serum LDL-C (reduction by $16 \%$ ) and the PCSK9 (inhibition by 33\%) is not surprising, as it has been found that variation in levels of the circulating PCSK9 can explain only $7-8 \%$ of the variation in levels of circulating LDL-C [30]. It is also worth noting that the main aim of this study was to evaluate the immunogenicity and safety of the L-IFPTA vaccine in the healthy state and in a model with close physiological similarity to humans.

The adjuvanted vaccine formulations may be accompanied with increased local and systemic reactions called reactogenicity [31,32]. Reactogenicity signifies a subset of adverse reactions that appear soon after vaccination and is a physical manifestation of the inflammatory response to vaccination, including redness, swelling or induration at the vaccination site, as well as systemic symptoms, such as malaise, fever, shivering or rash [32]. In this study, we observed no such local and systemic symptoms in vaccinated monkeys.

It is suggested that the immune-mediated responses and injection-related reactions are the main mechanisms behind the progression of adverse events after vaccination. Vaccination may provoke the localized and systemic inflammatory responses including a destructive elevation in acute-phase proteins and proinflammatory cytokines [32]. The well-known inflammatory markers commonly employed for monitoring systemic inflammation responses after vaccination are TNF- $\alpha$, IL-1, IL-6, and CRP [33]. At the injection site, the localized inflammatory response may be primed after vaccination due to the formation of antigen-antibody complexes and activation of the complement system, which rapidly induces inflammatory cells, such as tissue macrophages, to secrete pro-inflammatory cytokines, such as IL- $1 \alpha$, IL-1 $\beta$, IL-6, TNF, and interferon, into the circulation, resulting in the systemic inflammatory response, such as fever [32,34]. Acting either directly or indirectly on the specialized neurons of the thermoregulatory center of the hypothalamus, these cytokines promote the generation of E-series prostaglandins that increase the host's thermoregulatory set-point, leading to an elevation in the body temperature [34]. Moreover, systemic over-secretion of proinflammatory cytokines, together with reduced levels of anti-inflammatory cytokines, can result in systemic symptoms [34]. IL-1 and IL-6 have a central role in inducing the hepatic synthesis of the acute-phase proteins, such as CRP, in the liver. Notably, such inflammatory responses are mostly detectable in plasma or serum [33]. Our results indicated that the L-IFPTA vaccine exerted no significant effects on pro-inflammatory mediators (IL- $1 \alpha$, IL- $1 \beta$, IL- 6, TNF- $\alpha$, IFN- $\gamma$, and hs-CRP) and antiinflammatory cytokines (IL-4, IL-2, IL-8 and IL-10) in vaccinated monkeys. Furthermore, the MCP-1/VEGF/EGF pathway is involved in the migration and infiltration of inflam- 
matory monocytes into the vascular wall and thereby provokes the local inflammation at the vaccination site [35-37]. We found no significant changes in the serum levels of such inflammatory mediators in the vaccinated monkeys.

The imbalanced production of inflammatory cytokines can result in tissue and organ damage [34]. Thus, the toxic effect on vital organs is another important concern about the safety of vaccines. Blood circulating biomarkers that manifest the damage of the liver, kidneys, and thyroid glands are considered as sensitive indicators for toxicity. Vaccinated monkeys showed no significant changes in ALP, AST, ALT, and bilirubin values as markers of liver function; creatinine, urea, and uric acid values as markers of kidney function; and TSH value as the marker of thyroid function. However, the results of toxicity studies, which are currently ongoing by the present research group, can also further determine the safety of the L-IFPTA vaccine in the near future. These findings are further supported by a recent study that showed the antiPCSK9 vaccine not only did not damage the liver and kidneys but also markedly alleviated the renal lipid deposition and fibrosis and reduced the formation of fatty liver in hypercholesterolemic mice [17]. Moreover, no effects on food intake, body weight, and mortality were found in vaccinated monkeys, further supporting the safety of the antiPCSK9 L-IFPTA vaccine.

\section{Conclusions}

In summary, our findings indicate that the L-IFPTA vaccine is sufficiently immunogenic and safe in non-human primates. Therefore, this pre-clinical result suggests that the L-IFPTA vaccine might be an eligible candidate for phase I clinical trials in humans.

Author Contributions: Conceptualization, A.S. and A.A.M.-B.; methodology, A.A.M.-B., M.R.J., M.B. and A.S.; formal analysis, A.S.; investigation, A.M.G., H.S. and A.A.M.-B.; writing-original draft preparation, A.A.M.-B. and A.S.; writing—review and editing, M.B., A.M.G., M.R.J. and H.S.; supervision, A.S.; funding acquisition, A.S. All authors have read and agreed to the published version of the manuscript.

Funding: Research reported in this publication was supported by Elite Researcher Grant Committee under award number 963401 from the National Institutes for Medical Research Development (NIMAD), Tehran, Iran. We are also thankful for the support from Mashhad University of Medical Sciences, Mashhad, Iran (grant number: 971266).

Institutional Review Board Statement: The study was conducted according to the guidelines of the Declaration of Helsinki, and approved by the Institutional Ethics of Mashhad University of Medical Sciences, Mashhad, Iran (IR.MUMS.PHARMACY.REC.1397.113; approval date: 19 February 2019).

Informed Consent Statement: Not applicable.

Data Availability Statement: Data associated with this study are available from the corresponding author on a reasonable request.

Acknowledgments: We are thankful for the support from the Iran's National Elites Foundation, Tehran, Iran. This study was part of Amir Abbas Momtazi-Borojeni's post-doctoral fellowship at Iran's National Elites Foundation. We are thankful for the kind assistance of Sharam Rabbani in handling the animals during injection and sampling episodes.

Conflicts of Interest: The authors declare no conflict of interest.

\section{References}

1. Sabatine, M.S.; Giugliano, R.; Wiviott, S.D.; Raal, F.J.; Blom, D.J.; Robinson, J.; Ballantyne, C.M.; Somaratne, R.; Legg, J.; Wasserman, S.M.; et al. Efficacy and Safety of Evolocumab in Reducing Lipids and Cardiovascular Events. N. Engl. J. Med. 2015, 372, 1500-1509. [CrossRef]

2. Robinson, J.G.; Farnier, M.; Krempf, M.; Bergeron, J.; Luc, G.; Averna, M.; Stroes, E.S.; Langslet, G.; Raal, F.J.; El Shahawy, M.; et al. Efficacy and Safety of Alirocumab in Reducing Lipids and Cardiovascular Events. N. Engl. J. Med. 2015, 372, 1489-1499. [CrossRef] [PubMed]

3. Sabatine, M.S.; Giugliano, R.P.; Keech, A.C.; Honarpour, N.; Wiviott, S.D.; Murphy, S.A.; Kuder, J.F.; Wang, H.; Liu, T.; Wasserman, S.M.; et al. FOURIER Steering Committee and Investigators. Evolocumab and clinical outcomes in patients with cardiovascular disease. N. Engl. J. Med. 2017, 376, 1713-1722. [CrossRef] [PubMed] 
4. $\quad$ Nicholls, S.J.; Puri, R.; Anderson, T.; Ballantyne, C.M.; Cho, L.; Kastelein, J.J.; Koenig, W.; Somaratne, R.; Kassahun, H.; Yang, J.; et al. Effect of evolocumab on progression of coronary disease in statin-treated patients: The glagov randomized clinical trial. JAMA 2016, 316, 2373-2384. [CrossRef]

5. Momtazi-Borojeni, A.A.; Sabouri-Rad, S.; Gotto, A.M., Jr.; Pirro, M.; Banach, M.; Awan, Z.; Barreto, G.E.; Sahebkar, A. PCSK9 and inflammation: A review of experimental and clinical evidence. Eur. Heart J. Cardiovasc. Pharmacother. 2019, 5, 237-245. [CrossRef] [PubMed]

6. Catapano, A.; Papadopoulos, N. The safety of therapeutic monoclonal antibodies: Implications for cardiovascular disease and targeting the PCSK9 pathway. Atherosclerosis 2013, 228, 18-28. [CrossRef] [PubMed]

7. Do, R.Q.; Vogel, R.A.; Schwartz, G.G. PCSK9 Inhibitors: Potential in Cardiovascular Therapeutics. Curr. Cardiol. Rep. 2013, 15, 1-12. [CrossRef]

8. Hall, S.S. A gene of rare effect. Nature 2013, 496, 152-155. [CrossRef]

9. Sahebkar, A.; Watts, G.F. New Therapies Targeting apoB Metabolism for High-Risk Patients with Inherited Dyslipidaemias: What Can the Clinician Expect? Cardiovasc. Drugs Ther. 2013, 27, 559-567. [CrossRef]

10. Maningat, P.; Gordon, B.R.; Breslow, J.L. How Do We Improve Patient Compliance and Adherence to Long-Term Statin Therapy? Curr. Atheroscler. Rep. 2013, 15, 291. [CrossRef]

11. Kazi, D.S.; Moran, A.E.; Coxson, P.G.; Penko, J.; Ollendorf, D.A.; Pearson, S.D.; Tice, J.; Guzman, D.; Bibbins-Domingo, K. Cost-effectiveness of PCSK9 Inhibitor Therapy in Patients with Heterozygous Familial Hypercholesterolemia or Atherosclerotic Cardiovascular Disease. JAMA 2016, 316, 743-753. [CrossRef]

12. Marquina, C.; Zomer, E.; Vargas-Torres, S.; Zoungas, S.; Ofori-Asenso, R.; Liew, D.; Ademi, Z. Novel Treatment Strategies for Secondary Prevention of Cardiovascular Disease: A Systematic Review of Cost-Effectiveness. Pharmacoeconomics 2020, 38, 1095-1113. [CrossRef]

13. Bartelds, G.M.; Krieckaert, C.L.M.; Nurmohamed, M.T.; van Schouwenburg, P.; Lems, W.F.; Twisk, J.W.R.; Dijkmans, B.A.C.; Aarden, L.; Wolbink, G.J. Development of Antidrug Antibodies Against Adalimumab and Association with Disease Activity and Treatment Failure During Long-term Follow-up. JAMA 2011, 305, 1460-1468. [CrossRef]

14. Fattori, E.; Cappelletti, M.; Surdo, P.L.; Calzetta, A.; Bendtsen, C.; Ni, Y.G.; Pandit, S.; Sitlani, A.; Mesiti, G.; Carfí, A.; et al. Immunization against Proprotein Convertase Subtilisin-like/Kexin type 9 (PCSK9) lowers plasma LDL-cholesterol levels in mice. J. Lipid Res. 2012, 53, 1654-1661. [CrossRef] [PubMed]

15. Pan, Y.; Zhou, Y.; Wu, H.; Chen, X.; Zihua, Z.; Zhang, H.; Zhou, Z.; Qiu, Z.; Liao, Y. A Therapeutic Peptide Vaccine Against PCSK9. Sci. Rep. 2017, 7, 12534. [CrossRef] [PubMed]

16. Crossey, E.; Amar, M.J.; Sampson, M.; Peabody, J.; Schiller, J.T.; Chackerian, B.; Remaley, A.T. A cholesterol-lowering VLP vaccine that targets PCSK9. Vaccine 2015, 33, 5747-5755. [CrossRef] [PubMed]

17. Wu, D.; Zhou, Y.; Pan, Y.; Li, C.; Wang, Y.; Chen, F.; Chen, X.; Yang, S.; Zhou, Z.; Liao, Y.; et al. Vaccine Against PCSK9 Improved Renal Fibrosis by Regulating Fatty Acid $\beta$-Oxidation. J. Am. Heart Assoc. 2020, 9, e014358. [CrossRef]

18. You, S.; Guo, X.; Xue, X.; Li, Y.; Dong, H.; Ji, H.; Hong, T.; Wei, Y.; Shi, X.; He, B. PCSK9 Hapten Multicopy Displayed onto Carrier Protein Nanoparticle: An Antiatherosclerosis Vaccine. ACS Biomater. Sci. Eng. 2019, 5, 4263-4271. [CrossRef] [PubMed]

19. Galabova, G.; Brunner, S.; Winsauer, G.; Juno, C.; Wanko, B.; Mairhofer, A.; Lührs, P.; Schneeberger, A.; Von Bonin, A.; Mattner, F.; et al. Peptide-Based Anti-PCSK9 Vaccines-An Approach for Long-Term LDLc Management. PLoS ONE 2014, 9, e114469. [CrossRef]

20. Landlinger, C.; Pouwer, M.G.; Juno, C.; Van Der Hoorn, J.W.; Pieterman, E.J.; Jukema, J.W.; Staffler, G.; Princen, H.; Galabova, G. The AT04A vaccine against proprotein convertase subtilisin/kexin type 9 reduces total cholesterol, vascular inflammation, and atherosclerosis in APOE*3Leiden.CETP mice. Eur. Heart J. 2017, 38, 2499-2507. [CrossRef] [PubMed]

21. Momtazi-Borojeni, A.A.; Jaafari, M.R.; Badiee, A.; Sahebkar, A. Long-term generation of antiPCSK9 antibody using a nanoliposome-based vaccine delivery system. Atherosclerosis 2019, 283, 69-78. [CrossRef] [PubMed]

22. Momtazi, A.A.; Jaafari, M.R.; Badiee, A.; Banach, M.; Sahebkar, A. Therapeutic effect of nanoliposomal PCSK9 vaccine in a mouse model of atherosclerosis. BMC Med. 2019, 17, 223. [CrossRef]

23. Momtazi, A.A.; Nik, M.E.; Jaafari, M.R.; Banach, M.; Sahebkar, A. Effects of immunization against PCSK9 in an experimental model of breast cancer. Arch. Med. Sci. 2019, 15, 570-579. [CrossRef] [PubMed]

24. Momtazi-Borojeni, A.; Jaafari, M.R.; Abdollahi, E.; Banach, M.; Sahebkar, A. P704Nanoliposomal anti-PCSK9 vaccine ameliorates glucose intolerance and insulin resistance in diabetic rats. Eur. Heart J. 2019, 40. [CrossRef]

25. Momtazi-Borojeni, A.A.; Jaafari, M.R.; Afshar, M.; Banach, M.; Sahebkar, A. PCSK9 immunization using nanoliposomes: Preventive efficacy against hypercholesterolemia and atherosclerosis. Arch. Med. Sci. 2021, 17. [CrossRef]

26. Momtazi-Borojeni, A.; Jaafari, M.R.; Banach, M.; Sahebkar, A. P6194Therapeutic effect of nanoliposomal anti-PCSK9 vaccine on hypercholesterolemia and atherosclerosis in C57BL/ 6 mice. Eur. Heart J. 2019, 40. [CrossRef]

27. Momtazi-Borojeni, A.; Jaafari, M.R.; Badiee, A.A.; Banach, M.; Sahebkar, A.A. P6195Nanoliposomal anti-PCSK9 vaccine induces long-term and safe protection against atherosclerosis in C57BL/ 6 mouse. Eur. Heart J. 2019, 40. [CrossRef]

28. Schneeberger, A.; Mandler, M.; Otava, O.; Zauner, W.; Mattner, F.; Schmidt, W. Development of AFFITOPE vaccines for Alzheimer's disease (AD)—from concept to clinical testing. JNHA 2009, 13, 264-267. [CrossRef]

29. Banach, M.; Penson, P. What have we learned about lipids and cardiovascular risk from PCSK9 inhibitor outcome trials: ODYSSEY and FOURIER? Cardiovasc. Res. 2019, 115, e26-e31. [CrossRef] 
30. Lakoski, S.G.; Lagace, T.A.; Cohen, J.C.; Horton, J.D.; Hobbs, H.H. Genetic and Metabolic Determinants of Plasma PCSK9 Levels. J. Clin. Endocrinol. Metab. 2009, 94, 2537-2543. [CrossRef]

31. Di Pasquale, A.; Preiss, S.; Silva, F.M.D.O.E.; Garçon, N. Vaccine Adjuvants: From 1920 to 2015 and Beyond. Vaccines 2015, 3, 320-343. [CrossRef]

32. Hervé, C.; Laupèze, B.; Del Giudice, G.; Didierlaurent, A.M.; Da Silva, F.T. The how's and what's of vaccine reactogenicity. NPJ Vaccines 2019, 4, 39. [CrossRef] [PubMed]

33. Lim, P.W.; Garssen, J.; Sandalova, E. Potential Use of Salivary Markers for Longitudinal Monitoring of Inflammatory Immune Responses to Vaccination. Mediat. Inflamm. 2016, 2016, 1-12. [CrossRef] [PubMed]

34. Institute of Medicine (US); Committee to Review Adverse Effects of Vaccines; Stratton, K.R.; Clayton, E.W. Adverse Effects of Vaccines: Evidence and Causality; National Academies Press: Washington, DC, USA, 2012.

35. Lien, M.Y.; Chang, A.C.; Tsai, H.C.; Tsai, M.H.; Hua, C.H.; Cheng, S.P.; Wang, S.W.; Tang, C.H. Monocyte chemoattractant protein 1 promotes VEGF-A expression in OSCC by activating ILK and MEK1/2 signaling and downregulating miR-29c. Front. Oncol. 2020, 10, 2536. [CrossRef]

36. Mitchell, L.A.; Henderson, A.J.; Dow, S.W. Suppression of Vaccine Immunity by Inflammatory Monocytes. J. Immunol. 2012, 189, 5612-5621. [CrossRef] [PubMed]

37. Sahebkar, A.; Momtazi-Borojeni, A.A.; Banach, M. PCSK9 vaccine: So near, yet so far! Eur. Heart J. 2021. [CrossRef] 